\title{
Turbulence and waves in numerically simulated slope flows
}

\author{
Evgeni Fedorovich ${ }^{\mathrm{a}}$ And Alan Shapiro \\ School of Meteorology, Universtiy of Oklahoma, 120 David L. Boren Blvd., 73072 Norman, USA
}

Received 20 April 2009

\begin{abstract}
Direct numerical simulation (DNS) is applied to investigate properties of katabatic and anabatic flows along thermally perturbed (in terms of surface buoyancy flux) sloping surfaces in the absence of rotation. Numerical experiments are conducted for homogeneous surface forcings over infinite planar slopes. The simulated flows are the turbulent analogs of the Prandtl (1942) one-dimensional laminar slope flow. The simulated flows achieve quasi-steady periodic regimes at large times, with turbulent fluctuations being modified by persistent low-frequency oscillatory motions with frequency equal to the product of the ambient buoyancy frequency and the sine of the slope angle. These oscillatory wave-type motions result from interactions between turbulence and ambient stable stratification despite the temporal constancy of the surface buoyant forcing. The structure of the mean-flow fields and turbulence statistics in simulated slope flows is analyzed. An integral dynamic similarity constraint for steady slope/wall flows forced by surface buoyancy flux is proposed and quantitatively verified against the DNS data.
\end{abstract}

Key words: Katabatic / anabatic / numerical simulation / boundary layer / turbulence

Résumé - Turbulence et ondes dans la simulation numérique d'écoulements de pente. Les propriétés de vents catabatiques et anabatiques le long de pentes perturbées thermiquement (présence d'un flux de buoyancy de surface), en absence de rotation, sont étudiées par la voie de la simulation numérique directe. Les pentes considérées sont choisies planes et infinies. Le forçage à la paroi est toujours homogène. Les écoulements ainsi simulés sont les analogues turbulents de l'écoulement de pente laminaire et monodimensionnel de Prandtl (1942). Aux temps longs, un régime périodique quasi permanent s'installe. Les fluctuations turbulentes se trouvent alors modifiées par des mouvements oscillatoires persistants basse fréquence à une fréquence égale au produit de la fréquence de flottabilité de l'ambiance par le sinus de l'angle de la pente. Ces mouvements oscillatoires, similaires à des ondes, résultent en fait d'interactions entre la turbulence et la stratification ambiante, stable, et ce malgré le caractère stationnaire du forçage thermique pariétal. La structure des champs moyens et les grandeurs statistiques caractéristiques de la turbulence des écoulements obtenus sont analysées. Une loi de similitude dynamique intégrale est proposée pour les écoulements de pente/paroi permanents contraints par la présence d'un flux surfacique de flottabilité. Cette loi est validée quantitativement à l'aide des résultats de la simulation numérique directe.

Mots clés : Catabatique / anabatique / simulation numérique / couche limite / turbulence

\section{Introduction}

Slope winds (flows) are typical for vast areas of the Earth, and often play an important role in the weather and climate of these areas. From the standpoint of basic fluid dynamics, slope winds are buoyantly driven boundary-layer-type flows along heated or cooled sloping surfaces in a stratified fluid. It is common to distinguish between anabatic winds, which are driven by surface heating, and katabatic winds, which result from surface

\footnotetext{
a Corresponding author: fedorovich@ou.edu
}

cooling. Slope flows conflate three characteristic aspects of geophysical fluid dynamics: buoyant forcing, stratification, and turbulence. There are still many open questions regarding the structure and properties of these flows. Of particular interest for practical applications are the mean flow and turbulence structure of slope winds as functions of surface thermal forcing and slope angle.

An early milestone in the conceptual understanding of slope flows was the development of the Prandtl [2] one-dimensional model for a flow of a viscous stablystratified fluid along a uniformly cooled or heated sloping planar surface. Flow in the model has a boundary-layer 
character (low-level jet topped by weak reversed flow). The along-slope advection of environmental (mean) temperature balances thermal diffusion, and the along-slope component of buoyancy balances diffusion of along-slope momentum. Observations suggest that, with appropriately tuned parameters, this simple model provides a good description of the vertical structure of katabatic flows and a reasonable approximation of anabatic flows.

In the present study, the structural features of turbulent anabatic and katabatic flows along infinite planar slopes are investigated by means of direct numerical simulation (DNS). The DNS experiments explore prototypical physical effects in idealized downscaled atmospheric slope flows. Particular emphasis is placed on studying the dependence of the mean profiles and turbulence statistics in slope flows on the sign and magnitude of surface buoyancy forcing (in terms of surface buoyancy flux) and the slope steepness.

\section{Equations of slope-flow dynamics}

The momentum balance equations for a small-scale (very large Rossby number) flow in the Boussinesq approximation are the following:

$$
\begin{aligned}
\frac{\partial u}{\partial t}+u \frac{\partial u}{\partial x}+v \frac{\partial u}{\partial y}+w \frac{\partial u}{\partial z}= & -\frac{\partial \pi}{\partial x}+b \sin \alpha \\
& +\nu\left(\frac{\partial^{2} u}{\partial x^{2}}+\frac{\partial^{2} u}{\partial y^{2}}+\frac{\partial^{2} u}{\partial z^{2}}\right)
\end{aligned}
$$

$\frac{\partial v}{\partial t}+u \frac{\partial v}{\partial x}+v \frac{\partial v}{\partial y}+w \frac{\partial v}{\partial z}=-\frac{\partial \pi}{\partial y}+\nu\left(\frac{\partial^{2} v}{\partial x^{2}}+\frac{\partial^{2} v}{\partial y^{2}}+\frac{\partial^{2} v}{\partial z^{2}}\right)$

$$
\begin{aligned}
\frac{\partial w}{\partial t}+u \frac{\partial w}{\partial x}+v \frac{\partial w}{\partial y}+w \frac{\partial w}{\partial z}= & -\frac{\partial \pi}{\partial z}+b \cos \alpha \\
& +\nu\left(\frac{\partial^{2} w}{\partial x^{2}}+\frac{\partial^{2} w}{\partial y^{2}}+\frac{\partial^{2} w}{\partial z^{2}}\right)
\end{aligned}
$$

with the heat/buoyancy balance presented by

$$
\begin{aligned}
\frac{\partial b}{\partial t}+u \frac{\partial b}{\partial x}+v \frac{\partial b}{\partial y}+w \frac{\partial b}{\partial z}= & -N^{2}(u \sin \alpha+w \cos \alpha) \\
& +\nu_{h}\left(\frac{\partial^{2} b}{\partial x^{2}}+\frac{\partial^{2} b}{\partial y^{2}}+\frac{\partial^{2} b}{\partial z^{2}}\right)
\end{aligned}
$$

and mass conservation represented by the continuity equation for an incompressible fluid,

$$
\frac{\partial u}{\partial x}+\frac{\partial v}{\partial y}+\frac{\partial w}{\partial z}=0
$$

In the above equations, $u, v, w$ are velocity components in the right-hand slope-following Cartesian coordinate system $x$ (upslope), $y$ (cross-slope), $z$ (slope-normal), $\pi=\left[p-p_{e}\left(z^{\prime}\right)\right] / \rho_{r}$ is the normalized pressure perturbation, $\left[p_{e}\left(z^{\prime}\right)\right.$ is the environmental pressure, $z^{\prime}$ is the true vertical coordinate, $\rho_{r}=$ const is the reference density value], $b \equiv \beta \theta$ is the buoyancy with $\theta=\Theta-\Theta_{e}\left(z^{\prime}\right)$ being the potential temperature perturbation and $\beta=g / \Theta_{r}$ the buoyancy parameter $\left(\Theta_{r}=\right.$ const is the reference potential temperature value, $g$ is the gravity acceleration), $\gamma=\mathrm{d} \Theta_{e} / \mathrm{d} z^{\prime}=$ const is the gradient of environmental potential temperature, $\alpha$ is the slope angle, $\nu$ is the kinematic viscosity, $\nu_{h}$ is the thermal diffusivity, and $N=(\beta \gamma)^{1 / 2}$ is the Brunt-Väisälä (buoyancy) frequency.

The lateral boundary conditions for prognostic variables $(u, v, w, b)$ and normalized pressure $\pi$ are periodic (the sloping surface is supposed to be doubly-infinite along $x$ and $y$ ). The upper boundary conditions (large $z$ ) are $\partial \varphi / \partial z=0$, where $\partial \varphi / \partial z=0$ is any of $(u, v, w, b)$, and $\partial \pi / \partial z$ is obtained from $(3)$. The surface $(z=0)$ conditions are no-slip and impermeability $(u=v=w=0)$, with $\partial \pi / \partial z$ obtained from $(3)$, and $\nu_{h}(\partial b / \partial z)=-B_{s}$, where $B_{s}$ is the surface buoyancy flux which also has a meaning of the surface energy production rate.

\section{Numerical simulation of turbulent slope flows}

In this study, idealized turbulent anabatic and katabatic flows along double-infinite slopes have been investigated using direct numerical simulation (DNS), which implies resolving all scales of turbulent motion down to the viscous dissipation scale. The numerical algorithm employed to directly solve (1)-(5) with $\operatorname{Pr}=\nu / \nu_{h}=1$ has generally been the same as the one used to reproduce laminar $[3,4]$ and turbulent $[1,5]$ buoyantly driven flows along thermally perturbed vertical plates and sloping surfaces. The values of the surface buoyancy flux $B_{s}$ have been taken as $-0.3 \mathrm{~m}^{2} \mathrm{~s}^{-3},-0.5 \mathrm{~m}^{2} \mathrm{~s}^{-3}$ for the katabatic flow cases and $0.3 \mathrm{~m}^{2} \mathrm{~s}^{-3}, 0.5 \mathrm{~m}^{2} \mathrm{~s}^{-3}$ for the anabatic flow cases. Each flow type has been investigated with the slope angle $\alpha$ of $30^{\circ}$ and $60^{\circ}$. For comparison, data from the Fedorovich and Shapiro [1] study of turbulent flow along a vertical heated wall $\left(\alpha=90^{\circ}\right)$ are also considered.

Applying the Reynolds decomposition to the flow variables in the buoyancy balance equation (4) and averaging this equation over time and spatially over $x-y$ planes parallel to the slope, we obtain:

$$
-N^{2} \bar{u} \sin \alpha+\nu \frac{\partial^{2} \bar{b}}{\partial z^{2}}-\frac{\partial \overline{b^{\prime} w^{\prime}}}{\partial z}=0
$$

where primes signify deviations from the averages denoted by overbars. Integrating (6) over $z$ from 0 to $\infty$ and noting that both molecular and turbulent fluxes of the buoyancy vanish at $\infty$, we obtain an integral form of the buoyancy balance:

$$
V_{I} L_{I} \equiv \int_{0}^{\infty} \bar{u} \mathrm{~d} z=B_{s} /\left(N^{2} \sin \alpha\right)
$$


a)

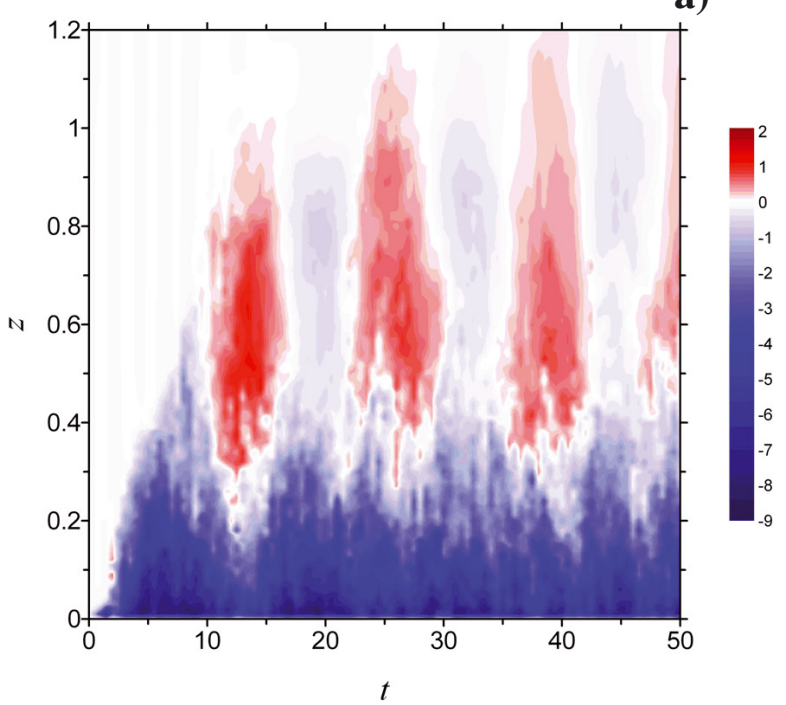

c)

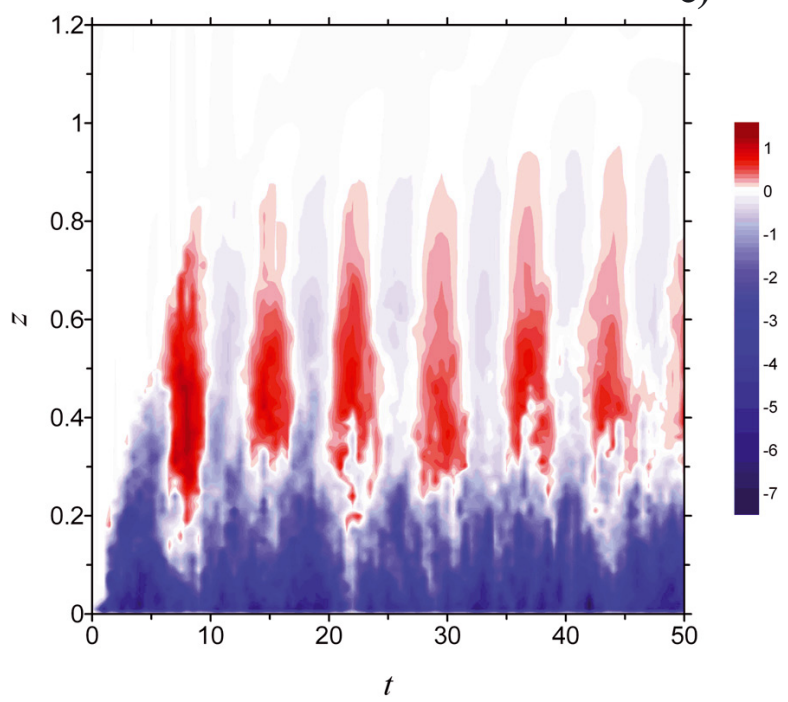

b)

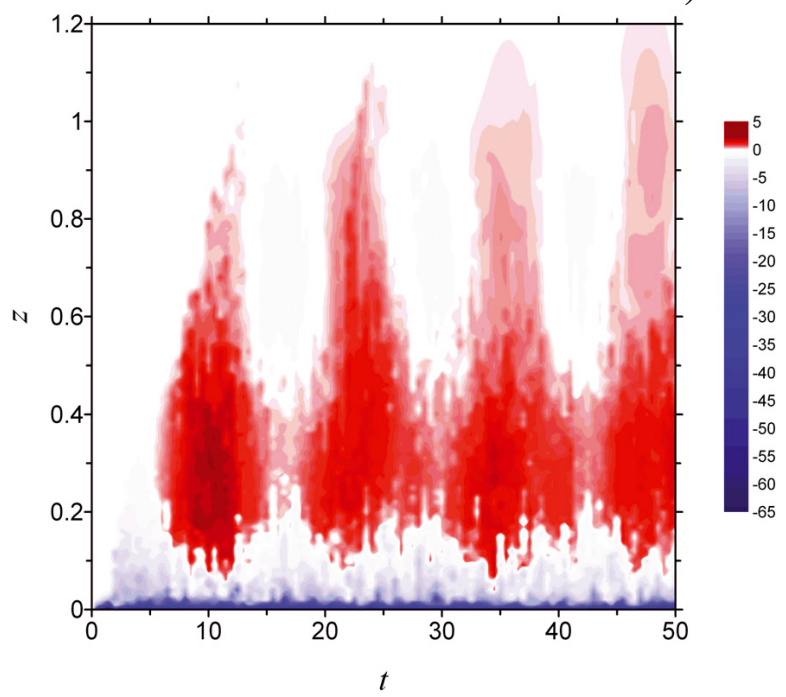

d)

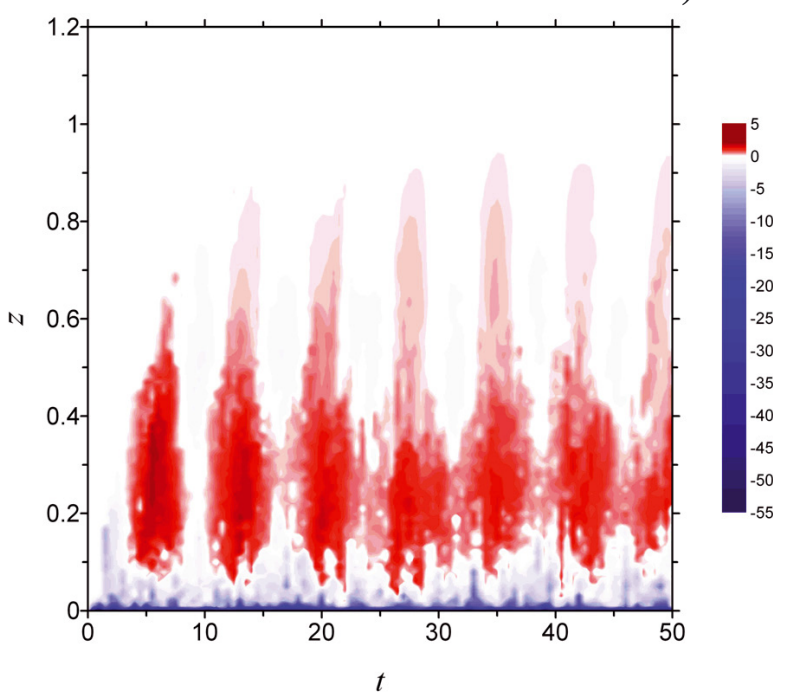

Fig. 1. Temporal variations of along-slope velocity component $\left(u\right.$, plots a and $\mathrm{c}$, in $\left.\mathrm{ms}^{-1}\right)$ and buoyancy $(b$, plots b and $\mathrm{d}$, in $\left.\mathrm{m} \mathrm{s}^{-2}\right)$ at different levels above the slope in the center of simulation domain for the katabatic flow cases $\left(B_{s}=-0.5 \mathrm{~m}^{2} \mathrm{~s}^{-3}\right.$, $\nu=10^{-4} \mathrm{~m}^{2} \mathrm{~s}^{-1}, N=1 \mathrm{~s}^{-1}$, resulting in $F i_{B}=-5000$ ) along slopes of different steepness: $30^{\circ}$ (plots a and b) and $60^{\circ}$ (plots $\mathrm{c}$ and $\mathrm{d})$.

where the product $V_{I} L_{I}$ is the volume flux. Note that we do not define either the integral velocity scale $V_{I}$ or the integral length scale $L_{I} \equiv \int_{0}^{\infty}\left(\bar{u} / V_{I}\right) \mathrm{d} z$. Based on the above considerations, one may introduce an integral slope-flow Reynolds number as:

$$
R e_{I} \equiv\left|V_{I} L_{I}\right| / \nu=\left|B_{s}\right|\left(\nu N^{2} \sin \alpha\right) \equiv\left|F p_{B}\right| / \sin \alpha
$$

where the flow forcing parameter $F p_{B} \equiv B_{s} \nu^{-1} N^{-2}$ is negative for a katabatic flow $\left(B_{s}<0\right)$ and positive for an anabatic flow $\left(B_{s}>0\right)$. The magnitude of $F p_{B}$ represents the ratio between the energy production at the surface and the work against buoyancy and viscous forces. From this definition of $R e_{I}$, we expect a particular slope flow to be more turbulent with increasing $\left|F p_{B}\right|$. In the performed DNS, values of $R e_{I}$ have been within the range of 3000 to 10000 .

\section{Results of DNS}

The spatial (in the $z$ direction) and temporal evolution of the simulated velocity ( $u$ component) and buoyancy $b$ fields in the central point of the $x-y$ plane is illustrated in Figure 1.

Results are shown for two katabatic flows with the same value of $F p_{B}=B_{s} \nu^{-1} N^{-2}=-5000$, but with different slope angles: $30^{\circ}$ and $60^{\circ}$. After passing through 

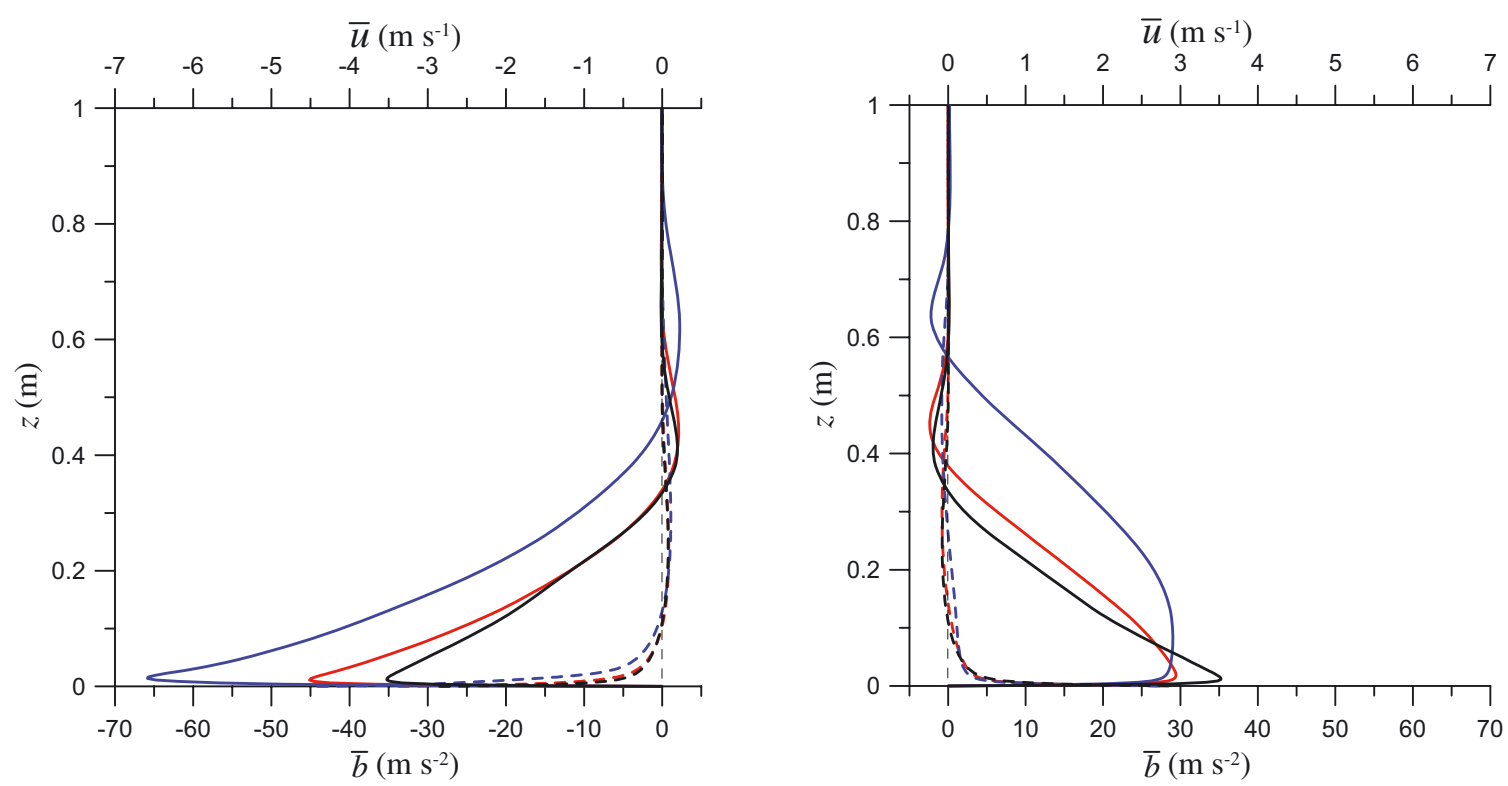

Fig. 2. Mean along-slope velocity ( $u$, solid lines) and buoyancy ( $b$, dashed lines) profiles in the katabatic (left) and anabatic (right) flows with $\left|B_{s}\right|=0.5 \mathrm{~m}^{2} \mathrm{~s}^{-3}, \nu=10^{-4} \mathrm{~m}^{2} \mathrm{~s}^{-1}$, and $N=1 \mathrm{~s}^{-1}\left(\left|F i_{B}\right|=5000\right)$ for three different slope angles: $30^{\circ}$ (blue lines), $60^{\circ}$ (red lines), and $90^{\circ}$ (black lines).
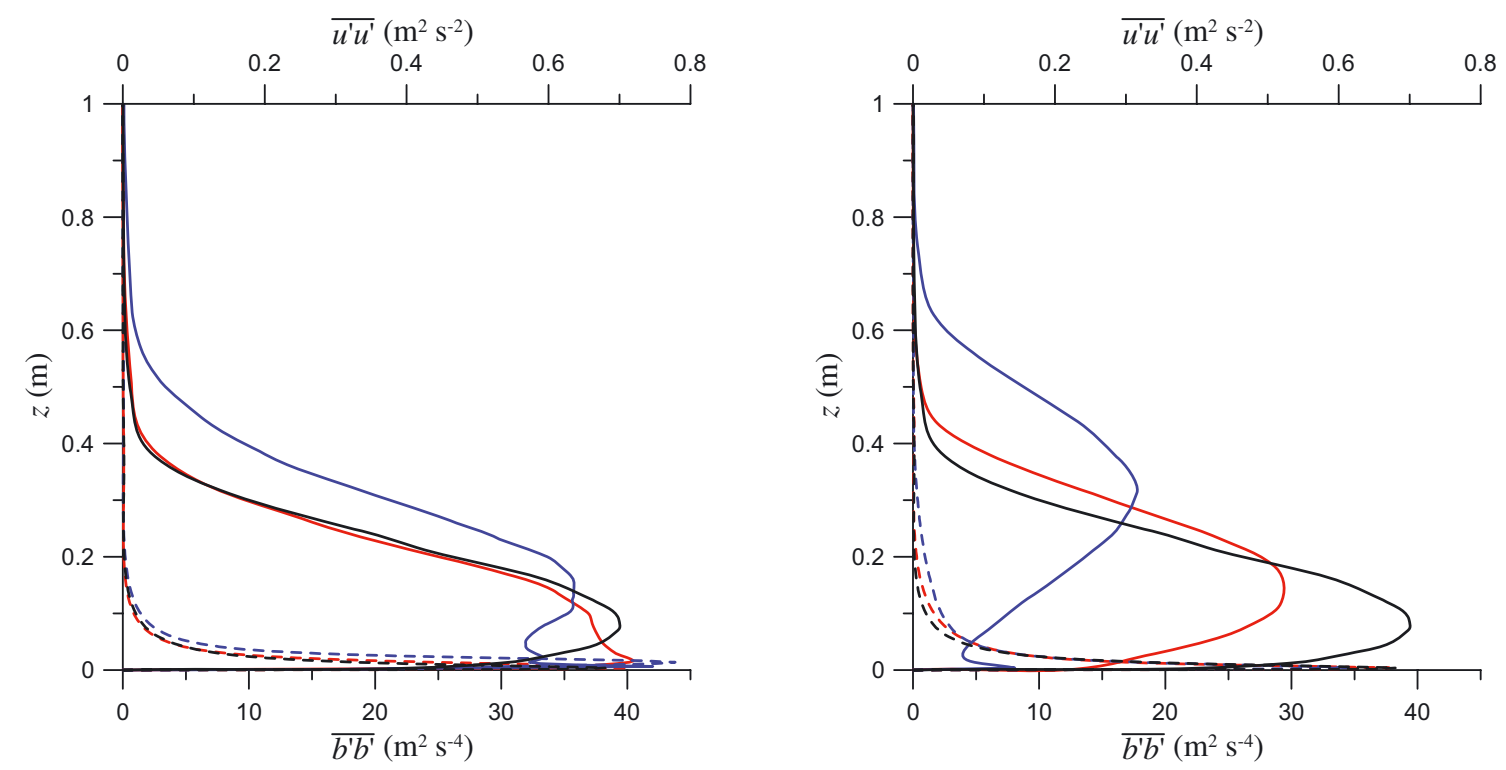

Fig. 3. Slope angle dependence of the along-slope velocity variances (solid lines) and buoyancy variances (dashed lines) in the katabatic (left) and anabatic (right) flows presented in Figure 2.

relatively short transition stages, both flows become turbulent and display random, large-amplitude fluctuations of velocity and buoyancy fields in the core regions. At larger distances from the slope, they show a quasi-periodic oscillatory behavior. Notably, only fluctuations with a frequency equal to the natural buoyancy frequency $N \sin \alpha$ in the environmental fluid dominate at large $z$. Fluctuations with other frequencies rapidly decay away from the slope beyond the turbulent core of the flow.

The mean velocity and buoyancy profiles presented in Figure 2 reveal a considerable sensitivity of the mean flow structure to the slope angle for both katabatic and anabatic flow cases. The mean profiles were obtained by averaging the simulated flow fields spatially over $x-y$ planes and temporally over at least 7 oscillation periods beyond the transition stage.

The shapes of the katabatic-flow velocity profiles for both slope angles $<90^{\circ}$ are very different from the shapes of velocity profiles in the anabatic flows. The differences in shape between the buoyancy profiles for both flow cases are less pronounced which is partially due to the fact that in both flow cases the buoyancy sharply drops (increases) in the very close vicinity of the wall. In the katabatic flow, stable environmental stratification in combination 
with negative surface buoyancy forcing lead to the effective suppression of vertical turbulent exchange in the flow region in the immediate vicinity of the slope. As a result, the mean velocity profile becomes jet-like, with a magnitude increases with decreasing slope angle, as also exhibited in the Prandtl [2] model.

Despite the obvious structural differences between the simulated katabatic and anabatic flows, the overall vertical extension of the flow in terms of the mean velocity appears to be almost the same for flows along slopes of the same angle. A direct evaluation of the mean velocity integrals in Figure 2 confirms the validity of (7) in these flow cases. Therefore, the combination $B_{s} /\left(N^{2} \sin \alpha\right)=$ $F p_{B} \nu / \sin \alpha$ of the governing parameters may be regarded as an integral dynamic similarity constraint for slope flows forced by the surface buoyancy flux. It should be noted that this criterion applies to both turbulent and laminar slope flows.

As revealed by the buoyancy variance profiles in Figure 3 , the buoyancy variances in both slope flow cases attain their maxima extremely close to the wall, within the region of maximum gradients in the mean buoyancy profiles (Fig. 2). The velocity variances of notable magnitudes are distributed over layers that are typically a few times thicker than the layers which contain most of the buoyancy variance. In both flow cases, narrow secondary maxima of $\overline{u^{\prime} u^{\prime}}$ in the close vicinity of the slope are observed. These secondary maxima, which are not found in the flow along a vertical wall $\left(\alpha=90^{\circ}\right)$, become more pronounced with decreasing slope angle and are more prominent in the katabatic flow.

\section{Summary}

Buoyantly driven slope flows along doubly-infinite cooled/heated inclined surfaces immersed in a stablystratified fluid were numerically simulated by means of DNS. The flows were driven by a spatially-uniform surface buoyancy flux $B_{s}$ and characterized by integral Reynolds numbers in the range from 3000 to 10000 . After the transition from a laminar to a turbulent phase, the simulated slope flows entered quasi-stationary oscillatory regimes with frequency of oscillations (associated with internal gravity waves) given by the product of the environmental Brunt-Väisälä frequency and sine of the slope angle, $N \sin \alpha$. Turbulent fluctuations gradually faded out with distance from the wall, while periodic oscillations persisted within the outer laminar flow regions before fading out. The slope-parallel velocity component variances consistently displayed secondary maxima very close to the wall, at distances comparable to those of the mean velocity maxima/minima. An integral dynamic similarity criterion, $\int_{0}^{\infty} \bar{u} \mathrm{~d} z=B_{s} /\left(N^{2} \sin \alpha\right)$, was derived from the averaged thermal energy equation and quantitatively verified against the DNS data.

\section{References}

[1] E. Fedorovich, A. Shapiro, Turbulent natural convection along a vertical plate immersed in a stably stratified fluid, J. Fluid Mech. (2009) to be published

[2] L. Prandtl, Führer durch die Strömungslehre, Vieweg und Sohn, Braunschweig 1942, pp. 382

[3] A. Shapiro, E. Fedorovich, Prandtl-number dependence of unsteady natural convection along a vertical plate in a stably stratified fluid. Int. J. Heat and Mass Transfer 47 (2004) 4911-4927

[4] A. Shapiro, E. Fedorovich, Natural convection in a stably stratified fluid along vertical plates and cylinders with temporally-periodic surface temperature variations, J. Fluid Mech. 546 (2006) 295-311

[5] A. Shapiro, E. Fedorovich, Coriolis effects in homogeneous and inhomogeneous katabatic flows, Q. J. R. Meteorol. Soc. 134 (2008) 353-370 